Proyecciones Journal of Mathematics

Vol. 29, No 2, pp. 145-164, August 2010.

Universidad Católica del Norte

Antofagasta - Chile

\title{
EXTREMALS OF A QUADRATIC COST OPTIMAL PROBLEM ON THE REAL PROJECTIVE LINE
}

\author{
V. $A Y A L A^{*}$ \\ J. C. RODRÍGUEZ ${ }^{\dagger}$ \\ UNIVERSIDAD CATÓLICA DEL NORTE, CHILE \\ and \\ L. A. B. SAN MARTÍN $\ddagger$ \\ UNIVERSIDADE ESTADUAL DE CAMPINAS \\ Received: July 2010. Accepted : August 2010
}

\begin{abstract}
Let $\Sigma$ be a bilinear control system on $\mathbf{R}^{2}$ whose matrices generate the Lie algebra $\mathbf{~} \mathbf{l}(2)$ of the Lie group $\mathrm{Sl}(2)$ : the group of order two real matrices with determinant 1 . In this work we focus on the extremals of a quadratic cost optimal problem for the angle system $\mathbf{P} \Sigma$ defined by the projection of $\Sigma$ onto the real projective line $\mathbf{P}^{1}$. It has been proved in [2] that through the Cartan-Killing form the cotangent bundle of $\mathbf{P}^{1}$ can be identified with a cone $\mathcal{C}$ in $\mathbf{~} \mathbf{l}(2)$. Via the Pontryagin Maximum Principle, we explicitly show the extremals by using the mentioned identification and the special form of the trajectories associated with the lifting of vector fields on $\mathbf{P} \Sigma$. We analyze both: the controllable case and when the system bf $P \Sigma$ give rise to control sets. Some examples are shown.
\end{abstract}

Subject classification : 35B50, 49J15, 93C15.

Key words : optimal control problem, extremals, Pontryagin maximum principle, bilinear control systems, angle system, Cartan-Killing form, real projective line, special linear Lie group.

\footnotetext{
${ }^{*}$ This research was partially supported by Proyectos FONDECYT No. 1100375 and Programa AMAZONAS SENIOR Processo Número:507/2007, FAPEAM.

${ }^{\dagger}$ Supported by Proyecto FONDECYT No. 3100137.

${ }^{\ddagger}$ Supported by CNPq grant No. 305513/2003-6 and FAPESP grant No. 07/06896-5
} 


\section{Introduction}

Optimal control problems with quadratic cost have already been analyzed with great success for linear control systems, see [6]. However, a great number of applications are modelled by bilinear dynamics. For instance, different classes of biological and chemical processes has been analyzed in [5], [8]. On the other hand, the state of the art of this theory is far from a sufficient understanding and require new approaches to meet the challenges in this field, see [4] for a nice exposition of control systems on fiber bundles. This paper analyze the quadratic cost optimal problem for a class of systems induced by a bilinear one.

Let $\Sigma$ be a bilinear control system on $\mathbf{R}^{2}$ whose matrices generate the Lie algebra $\mathbf{s l}(2)$ of the Lie group $\mathrm{Sl}(2)$ : the group of order two real matrices with determinant 1 . Precisely, $\Sigma$ is determined by the family of differential equations:

$$
\Sigma: \quad \dot{x}(t)=(A+u B) x(t), \quad t \in \mathbf{R}, x(t) \in \mathbf{R}^{2}
$$

Here, $A, B \in \mathbf{s l}(2)$, and $u$ is an element of the admissible restricted controls class $\mathrm{U}=\{u: \mathbf{R} \rightarrow[-1,1], u$ locally integrable $\}$.In this work we focus on the extremals of the quadratic cost optimal problem

$$
J=\min _{u(\cdot) \in[-1,1]} \frac{1}{2} \int_{0}^{T} u^{2} d t
$$

for the angle system $\mathbf{P} \Sigma$ defined by the projection of $\Sigma$ onto the real projective line $\mathbf{P}^{1}$, as follows

$$
\mathbf{P} \Sigma: \quad \dot{s}(t)=h(A, s(t))+u(t) h(B, s(t)), \quad s \in \mathbf{P}^{1}
$$

The projected dynamics on the sphere is induced by the formula

$$
h(X, s)=\left(X-\left(s^{\top} X s\right) I d\right) s
$$

with $I d$ the identity matrix, $u$ an element of the restricted admissible control set and $s^{\top} X s$ corresponds to the radial component of the vector field $X s$ and ()$^{\top}$ denotes transpose.

In other words, given two points $p$ and $q$ in $\mathbf{P}^{1}$ our aim is to find a trajectory of $\mathbf{P} \Sigma$ starting on $p$ and ending on $q$ minimizing the functional $J$ over all such a curves. 
It has been proved in [2] that through the Cartan-Killing form, the cotangent bundle of $\mathbf{P}^{1}$ can be identified with a cone $\mathcal{C}$ in $\operatorname{sl}(2)$. Via the Pontryagin Maximum Principle, we explicitly show the extremals by using the mentioned identification and the special form of the trajectories associated with the lifting of vector fields on $\mathbf{P} \Sigma$ to the cone. We analyze both: the controllable case and when the system $\mathbf{P} \Sigma$ give rise to control sets.

Since the projective real line is a compact manifold, it follows that given any two arbitrary points in $\mathbf{P}^{1}$ there exists an optimal path connecting them.

Through the paper we assume that $\Sigma$ satisfy the Lie algebra rank condition, $(L A R C)$, which means that the Lie algebra generated by $A$ and $B$ coincides with $\mathbf{s l}(2)$. Furthermore, we follow the references [1] and [3] which give an algebraic and geometric condition to the controllability of $\mathbf{P} \Sigma$, respectively.

The paper is organized as follows. Section 2 contains the Hamiltonian formalism on the cone in $\mathbf{s l}(2)$ and the trajectories associated with the lifting of vector fields on $\mathbf{P} \Sigma$. In Section 3 we describe the associated Hamiltonian and the explicitly form of the optimal control. In Section 4 we analyze the synthesis of the problem. We also give examples.

\section{Hamiltonian Formalism and Trajectories on $\mathcal{C}$}

In order to have an appropriated frame for the understanding of our problem, we first recall some results established by the authors in [2].

Let $G$ be a Lie group and $H \subset G$ a closed Lie subgroup. We consider the homogeneous space $G / H=\{z H: z \in G\}$. Denotes by $\mathbf{g}$ and $\mathbf{h}$ the Lie algebras of $G$ and $H$ respectively. Each $X \in \mathbf{g}$ induces a vector field $\widetilde{X}$ on $G / H$ and its flow is given by $\widetilde{X}_{t}(x)=\exp (t X) x, x \in G / H$.

If $X$ is a vector field on a differentiable manifold $N$, there exists a lifting $X^{*}$ of $X$ on the cotangent bundle $T^{*} N$. Moreover, $X^{*}$ is a Hamiltonian vector field and the corresponding Hamiltonian function $\mathcal{H}_{X^{*}}: T^{*} N \rightarrow \mathbf{R}$ is defined by

$$
\mathcal{H}_{X^{*}}(\alpha)=\alpha(X(\pi(\alpha))), \quad \alpha \in T^{*} N
$$

Here, $\pi: T^{*} N \rightarrow N, \alpha \longmapsto \pi(\alpha)$ is the fiber bundle projection.

As has been showed in [2], the elements of the cotangent bundle $T^{*}(G / H)$ can be represented by elements of $T^{*} G$. We specialize this identification to semisimple Lie groups. Through the Cartan-Killing form $\mathcal{K}$ the Lie algebra 
$\mathbf{g}$ of $G$ is identified with its dual $\mathbf{g}^{*}$ (see [10]). Let $X \in \mathbf{g}$ and $\widetilde{X}$ its induced vector field on the homogeneous space $G / H$. We have

Proposition 2.1. Let $\mathbf{g}$ be a semisimple Lie algebra and $\mathbf{h} \subset \mathbf{g}$ the Lie algebra of $H$. Then, the lifted vector field $\widetilde{X}^{*}$ on $T^{*}(G / H)$ induced by $X \in \mathbf{g}$ is given by

$$
\widetilde{X}^{*}(Y)=\operatorname{ad}(X)(Y) \text { with flow } \widetilde{X}_{t}^{*}(Y)=\operatorname{Ad}(\exp t X) Y .
$$

On the other hand, it is well known that in the semisimple Lie algebra $\operatorname{sl}(2)$, the form $\mathcal{K}$ is a multiple of the trace form. Precisely,

$$
\mathcal{K}(X, Y)=k \operatorname{tr}(X Y)=k\langle X, Y\rangle
$$

where $k \neq 0$ is a constant. In [1] is showed that the zeros set

$$
\mathcal{C}=\{X \in \mathbf{s l}(2): Q(X)=0\}
$$

of the corresponding quadratic form $Q(X)=\langle X, X\rangle=\operatorname{tr}\left(X^{2}\right)$ is a cone with respect to the basis

$$
H=\left(\begin{array}{rr}
1 & 0 \\
0 & -1
\end{array}\right), S=\left(\begin{array}{ll}
0 & 1 \\
1 & 0
\end{array}\right), R=\left(\begin{array}{rr}
0 & -1 \\
1 & 0
\end{array}\right)
$$

of $\mathbf{s l}(2)$. In fact, in the $\left(z_{1}, z_{2}, z_{3}\right)$-coordinates the equation of $\mathcal{C}$ with respect to $(2.1)$ is given by $z_{1}^{2}+z_{2}^{2}=z_{3}^{2}$.

The set $\mathcal{C}-\{0\}$ has two connected components, we distinguish them by putting $\mathcal{C}^{+}$and $\mathcal{C}^{-}$for the one which contains the matrix

$$
\left(\begin{array}{ll}
0 & 1 \\
0 & 0
\end{array}\right) \quad\left(\begin{array}{cc}
0 & 0 \\
-1 & 0
\end{array}\right)
$$

respectively. Just observe that the rotation group turns around $\mathcal{C}^{+}$while the group of diagonal matrices is transitive along the ray of upper triangular matrices in $\mathcal{C}^{+}$. Thus, $\mathrm{Sl}(2)$ acts transitively on $\mathcal{C}^{+}$. Furthermore, the action of $\mathrm{Sl}(2)$ into the set $\left[\mathcal{C}^{+}\right]$of rays of $\mathcal{C}^{+}$is equivalent to the action of $\mathrm{Sl}(2)$ in the projective line $\mathbf{P}^{1}$. We denote by $\mathcal{C}_{\text {int }}^{+}, \mathcal{C}_{\text {ext }}^{+}$the regions of $\mathbf{R}^{3}$ inside and outside the cone, respectively. In [2] an algebraic picture of the cone is given as follows 
Proposition 2.2. The entire cone $\mathcal{C}$ is identified with the cotangent bundle $T^{*}\left(\mathbf{P}^{1}\right)$.

For $P \in \mathcal{C}^{+}$the tangent plane $T_{P} \mathcal{C}^{+}$of $\mathcal{C}^{+}$at $P$ is

$$
T_{P} \mathcal{C}^{+}=\{W \in \mathbf{s l}(2):\langle P, W\rangle=0\} .
$$

Notice that any $Z \in \mathbf{s l}(2)$ defines, through the linear application ad $Z$ in sl (2), a linear differential equation in $\mathbf{s l}(2)$, explicitly given by $\dot{P}=[Z, P]$, $P \in \mathbf{s l}(2)$, whose trajectories are

$$
\exp (t \operatorname{ad} Z) P=A d(\exp t Z)(P), \text { with } P \in \mathbf{s l}(2) \text { and } t \in \mathbf{R} .
$$

In particular, $Z \in \mathbf{s l}(2)$ induces also a differential equation on $\mathcal{C}^{+}$. Just observe that $<P=[Z, P]>=0$.In the sequel, we will describe these trajectories in $\mathcal{C}^{+}$as the intersections of $\mathcal{C}^{+}$with the planes

$$
Z^{\perp}=\left\{P \in \mathcal{C}^{+}:\langle P, Z\rangle=c\right\}
$$

orthogonal to $Z$ with respect to the trace form.

Remark 2.1. In practice, with respect to the trace form, the plane orthogonal to any matrix of trace zero can be seen with the aid of the inner product $(\cdot, \cdot)$ in $\mathbf{s l}(2)$ which is defined by

$$
(P, W)=\left\langle P, W^{t}\right\rangle=\operatorname{tr}\left(P W^{\top}\right) .
$$

Actually, if we denote by $Z^{(\perp)}$ the orthogonal plane to $Z$ with respect to $(\cdot, \cdot)$, then $Z^{\perp}=\left(Z^{(\perp)}\right)^{\top}$. On the other hand, transposition is obtained by a reflection through the plane $\mathbf{s}$ of symmetric matrices. Therefore, $Z^{\perp}$ is the reflection through $\mathbf{s}$ of the plane orthogonal to $Z$ with respect to $(\cdot, \cdot)$. The authors in [1] give the following description of the trajectories on $\mathcal{C}$.

Proposition 2.3. According to the location of $Z$ respect to the cone, the trajectories of $\dot{P}=[Z, P]$ in $\mathcal{C}^{+}$are:

1. Ellipses around $\mathcal{C}^{+}$, if $Z \in \mathcal{C}_{\text {int }}$

2. Points in the ray of $\mathcal{C}^{+}$orthogonal to $Z$ or the parabolas $\{\langle Z, P\rangle=$ c) $\cap \mathcal{C}^{+}$, if $Z \in \mathcal{C}^{+}$

3. The two rays in $Z^{\perp} \cap \mathcal{C}^{+} \backslash\{0\}$ or the semi-hyperbolas $\{\langle Z, P\rangle=c\} \cap \mathcal{C}^{+}$, if $Z \in \mathcal{C}_{\text {ext }}$. 


\section{The Hamiltonian and the Optimal Control}

In this section, we establish the Pontryagin Maximum Principle $(P M P)$ for the angle system $\mathbf{P} \Sigma$ on $\mathbf{P}^{1}$ determined by a bilinear control system $\Sigma$ in the plane. From the principle, we characterize the optimal control for the quadratic cost.

The dynamic here described is determined by the bilinear control system (1.1), where $A$ and $B$ are two matrices of trace zero, with running cost (1.2) and bounded control $|u| \leq 1$.

For our purposes, the suitable version of the $P M P$ provided in [7], gives a first order necessary conditions for the optimality of quadratic control problems. In our particular case the principle reads:

Theorem 3.1. If $u_{*}$ is an optimal control and $x_{*}$ is the associated trajectory of $\mathbf{P} \Sigma$ on $\mathbf{P}^{1}$, there exists a constant $\lambda_{0} \geq 0$ and an absolutely continuous function $\lambda:[0, T] \longrightarrow T^{*} \mathbf{P}^{1}$, such that for almost every $t \in \operatorname{Dom}\left(x_{*}\right)$, $\left(\lambda, \lambda_{0}\right)$ never vanishing and the adjoint equation,

$$
\dot{\lambda}=-\lambda\left(A+u_{*} B\right)
$$

is satisfied. Furthermore, the optimal control $u_{*}(t)$ minimizes the Hamiltonian

$$
\mathcal{H}=\lambda(A+u B) x+\frac{1}{2} \lambda_{0} u^{2}
$$

on $[-1,1]$, through the curve $\left(\lambda(t), x_{*}(t)\right)$.

The pair $\left(u_{*}, x_{*}\right)$ is called extremal pair if $u_{*}$ is an admissible control and the corresponding trajectory $x_{*}$ satisfy the $P M P$ conditions. We normalize $\lambda_{0}$ to 1 .

By Proposition 2.2, the form of the Hamiltonian $\mathcal{H}$ and by using the invariance of the Cartan-Killing form $\mathcal{K}$, it turns out that the equation (3.1) can be rewritten as

$$
\frac{d P(t)}{d t}=[A+u B, P(t)], P(t) \in \mathcal{C}^{+}
$$

An admissible constant control $u$ induces the vector field $X^{u}=A+u B \in$ g. On the other hand, since that the cotangent bundle $T^{*} \mathbf{P}^{1}$ is identified 
with the cone $\mathcal{C}$, each curve $[0, T] \longrightarrow T_{z H}^{*} \mathbf{P}^{1}$ given by the $P M P$ is identified with a curve in $\mathcal{C}^{+}$. We obtain: the Hamiltonian for the quadratic optimal control problem

$$
\begin{gathered}
\mathcal{H}_{\widetilde{X}^{u}}: \mathcal{C}^{+} \rightarrow \mathbf{R} \text { is defined by } \\
\mathcal{H}_{\widetilde{X}^{u}}(P)=\langle P, A+u B\rangle+\frac{1}{2} u^{2}, P \in \mathcal{C}^{+}
\end{gathered}
$$

where $\widetilde{X}^{u}$ is the lifted vector field on $\mathcal{C}^{+}$induced by $X^{u}$ on $\mathbf{P}^{1}, u(\cdot) \in$ $[-1,1]$.

The Hamiltonian $\mathcal{H}$ is strictly convex in the control $u$ and its minimum over $[-1,1]$ is attained with

$$
\varphi(t)=-\langle P(t), B\rangle
$$

In particular, the optimal control $u_{*}$ minimizing $\mathcal{H}$ over $[-1,1]$ is determined by

$$
\mathrm{u}_{*}(t)=\left\{\begin{array}{lll}
1 & \text { if } & \varphi(t) \geq 1 \\
\varphi(t) & \text { if } & \varphi(t) \in(-1,1) \\
-1 & \text { if } & \varphi(t) \leq-1
\end{array}\right.
$$

We call the function $\varphi(t)$ the switching function of the control. Thus, the application of the PMP to any control system lead controls belonging to the interior or to the boundary of the control range $[-1,1]$.

\section{The Quadratic Optimal Control Synthesis}

In this section we analyze, on the real projective line, the extremals for the quadratic cost. These trajectories are obtained by the radial projection of the extremals in $\mathcal{C}^{+}$defined by the adjoint system (3.2). Precisely, given $p$ and $q$ two arbitrary points on $\mathbf{P}^{1}$ our goal is to find the extremals of the quadratic cost connecting these two points. Since in our case, the number of extremals are finite, it turns out that it is possible to find the optimal path in a closed analytic form. In future research, we hope to apply numerical algorithms to approach the optimal solutions.

We identify $\mathbf{P}^{1}$ with the ordered circle $\left\{e^{i \psi}:-\pi / 2<\psi \leq \pi / 2\right\}$. According to the shape of the optimal controls determined by the $P M P$ we need to consider the planes $\langle P, B\rangle=0,\langle P, B\rangle= \pm 1$ and the intersection 
of these planes with $\mathcal{C}^{+}$. We have the following relative positions. Namely,

The synthesis of the optimal control will be established by considering the following cases

Case $1\langle P, B\rangle=0$ doesn't meet the cone. In this case, $\langle P, B\rangle=1$ or $\langle P, B\rangle=$ -1 intersects $\mathcal{C}_{\text {int }}^{+}$but no both.

Case $2\langle P, B\rangle=0$ is tangent to the Cone $\mathcal{C}^{+}$. As the previous case, just one plane will meet the cone

Case $3\langle P, B\rangle=0$ divides the Cone $\mathcal{C}^{+}$in two regions. So, the planes $\langle P, B\rangle= \pm 1$ meet $\mathcal{C}_{\text {int }}^{+}$, see Figure 4.1 .

Figure 4.1: Intersection planes $\langle P, B\rangle=0, \pm 1$ with the cone $\mathcal{C}^{+}$

According to the proposition 2.3, if $Z \in \mathcal{C}_{\text {ext }}^{+}$the trajectories of the adjoint equation (3.2) are the rays in $Z^{\perp} \cap \mathcal{C}^{+} \backslash\{0\}$ or the hyperbolas $\{\langle Z, P\rangle=c\} \cap \mathcal{C}^{+}$. Recall that the rays are determined by the corresponding eigenvectors of the matrices.

We denote by $l_{+1}^{+}$and $l_{+1}^{-}$the rays of $Z=A+B$ and by $l_{-1}^{-}$and $l_{-1}^{+}$ those for $Z=A-B$. The intersection between $\mathcal{C}^{+}$and the orthogonal plane to $B$ are two lines denoted for $l_{0}^{1}$ and $l_{0}^{2}$. On $\mathbf{P}^{1}$ the projections of $l_{+1}^{-}, l_{+1}^{+}$, $l_{-1}^{-}, l_{-1}^{+}, l_{0}^{1}$ and $l_{0}^{2}$ are denoted by $q_{+1}^{-}, q_{+1}^{+}, q_{-1}^{-}, q_{-1}^{+}, q_{0}^{1}$ and $q_{0}^{2}$ respectively. Here $q_{+1}^{-}$and $q_{-1}^{-}$are attractors and $q_{+1}^{+}, q_{-1}^{+}$repellers. Without loss of generality, through the whole paper we assume $q_{-1}^{-}<q_{-1}^{+}$. The case $q_{-1}^{+}$ $<q_{-1}^{-}$is analogous. 
For $Z \in \mathbf{s l}(2)$, the linear differential equation $\dot{P}=[Z, P]$ on sl (2) induces a vector field on $\mathcal{C}^{+}$. Proposition 2.3 gives the form of the trajectories on $\mathcal{C}^{+}$and their projection on $\mathbf{P}^{1}$ are as follows: Ellipses onto the circle, points onto singularities, parabolas onto segments, lines onto singularities and semi-hyperbolas onto segments.

Via the adjoint action of $\mathrm{Sl}(2, \mathbf{R})$ on the cone, any rotation on $\mathcal{C}^{+}$is conjugated to the matrix

$$
R=\left(\begin{array}{rr}
0 & -1 \\
1 & 0
\end{array}\right) .
$$

In particular, they move in the counterclockwise direction.

In the next sections the synthesis of the optimal problem is established according to the relative position of the planes $\langle P, B\rangle=0, \pm 1$ and $\langle P, A \pm B\rangle=0$.

\subsection{The Controllable Case}

Through this section we assume that the projected control system (1.3) is controllable. Since the projective real line is a compact manifold, it follows that given any two arbitrary points in $\mathbf{P}^{1}$ there exists an optimal path connecting them and this path is coming from the Pontryagin Maximum Principle.

Theorem 5.2 in [1] gives an algebraic characterization of the controllability property of (1.1). From a geometric point of views, this result says that the bilinear control system is controllable in $\mathbf{R}^{2} \backslash\{0\}$ if and only if the segment

$$
A+u B:-1 \leq u \leq 1, \text { intercepts } \mathcal{C}_{\text {int }}^{+} .
$$

Of course, if (1.1) is controllable then the projected control system (1.3) is also controllable, see for instance [9].

Proposition 4.1. If $B \in \mathcal{C}_{i n t}^{+}$and the system $\Sigma$ is controllable then $A+$ $B \in \mathcal{C}_{\text {int }}^{+}$.

Proof: Since the system $\Sigma$ is controllable, from Theorem 5.2 in [1] it follows that the segment $A+u B:-1 \leq u \leq 1$, intercepts $\mathcal{C}_{\text {int }}^{+}$. So, there exists $u_{0} \in[-1,1]$ such that $A+u_{0} B$ belong to $\mathcal{C}_{\text {int }}^{+}$. Hence, the segment $A+B \in \mathcal{C}_{\text {int }}^{+}$because $B \in \mathcal{C}_{\text {int }}^{+}$. 
Remark 4.1. If $\Sigma$ is controllable and $-B \in \mathcal{C}_{\text {int }}^{+}$according to Proposition 4.1 $A-B \in \mathcal{C}_{\text {int }}^{+}$and the synthesis is analogous.

\subsubsection{Case 1. $\langle P, B\rangle=0$ does not meet $\mathcal{C}^{+}$}

Let us assume $B \in \mathcal{C}_{\text {int }}^{+}$. We follows the synthesis by the relative position of the planes $\langle P, B\rangle= \pm 1$ with the cone.

Suppose the plane $\langle P, B\rangle=1$ intersect the cone $C^{+}$.

We obtain two regions, the lower and the upper region. See Figure 4.2

$$
L=\left\{Z \in \mathcal{C}^{+} \mid 0<\langle Z, B\rangle<1\right\}, U=\left\{Z \in \mathcal{C}^{+} \mid\langle Z, B\rangle>1\right\}
$$

Figure 4.2: Intersection of the plane $\langle P, B\rangle=1$ with the cone $\mathcal{C}^{+}$

We claim: given two arbitrary points $p, q \in \mathbf{P}^{1}$ show an optimal path connecting them. In fact,

We have the possibilities:

1. $A+B \in \mathcal{C}_{\text {int }}^{+}$and $A-B \in \mathcal{C}_{\text {ext }}^{+}$

The trajectories of the adjoint system $(3.2)$ on $\mathcal{C}^{+}$induced by the bang control $u_{*}(t)=1$ are ellipses which we denote by $\varepsilon^{+}$. Denote by $\beta^{\varphi}$ the trajectories of the adjoint system for $u_{*}(t)=\varphi(t) \in(-1,1)$. 
Let $p \in \mathbf{P}^{1}$ and $\zeta_{0}$ an arbitrary point of the fiber of $p$, i.e., $\pi\left(\zeta_{0}\right)=p$, where $\pi$ denotes the radial projection from $\mathcal{C}^{+}$to $\mathbf{P}^{1}$. According to the $P M P$, the synthesis state as follows:

1.1. If $\zeta_{0} \in \pi^{-1}(p)$ lies on a ellipse $\varepsilon^{+}$contained in $U$ the initial condition $\zeta_{0}$ moves under the influence of $u_{*}=1$ (counterclockwise) until the intersection of $\varepsilon^{+}\left(\zeta_{0}\right)$ with the fiber of $\pi^{-1}(q)$. So, $\pi\left(\varepsilon^{+}\right)$is the optimal path.

1.2. Assume $\zeta_{0} \in U$ belongs to $\varepsilon^{+}$which intersects the plane $\langle P, B\rangle=1$. Let us assume that the fiber of $\pi^{-1}(q)$ intersects $\varepsilon^{+}$at the point $\xi$

a) If $\xi \in c l U$, and the control $u=1$ connect $\zeta_{0}$ with $\xi$. Then, as in the previous case the projection of the dynamic determined by $u_{*}=1$ gives the optimal path.

b) If $\xi \in L$, then $\zeta_{0}$ moves under the influence of $u_{*}=1$ up to the intersection with the plane $\langle P, B\rangle=1$. From this intersection point, it follows the trajectory corresponding to the differential equation induced by the control $\varphi(t)=-\langle P(t), B\rangle$ moving on $L$ until intersects the fiber of $q$. The projection of $\beta^{\varphi} \circ \varepsilon^{+}$gives rise the optimal path connecting them, starting on $p$ and ending on $q$.

c) If $\xi \in c l U$, and the control $u=1$ connect $\xi$ with $\zeta_{0}$, we follows the path $\beta^{\varphi} \circ \varepsilon^{+}$up to the second intersection with the plane $\langle P, B\rangle=1$. After that we use again $u=1$ to reach $\zeta_{0}$. So, the projection of the absolutely continuous curve $\varepsilon^{+} \circ \beta^{\varphi} \circ \varepsilon^{+}$.

1.3. If we start on $\zeta_{0} \in \operatorname{cl}(L)$,

a) Let $\xi \in \operatorname{cl}(L)$. Eventually, we could reach $q$ from $p$ as follows: through the projection of the differential equation determined by the optimal control $\varphi(t)=-\langle P(t), B\rangle \in(-1,1)$ with initial condition $\zeta_{0}$ and ending point $\xi$. Or, starting with the control $\varphi(t)=-\langle P(t), B\rangle$ up to the curve meets the plane $\langle P, B\rangle=1$ at the point $\eta$, to continue with a particular class of ellipses $\varepsilon^{+}$. Depending on the class, $\eta$ could be move towards $L$ or $U$. In fact, in the first case, the curve will reach $\xi$ inside of $L$,i.e., the positive direction of the ellipses (derivative at the point $\eta$ point out towards $L$ ). In the second case, the curve will continue with the ellipse up to meet the fiber of $q$. The projection of the last two mentioned curves will give other extremals.

b) If $\xi \in U$, we first need to meet the plane $\langle P, B\rangle=1$ at $\eta$ with an appropriate $\beta^{\varphi}$. Then, to continue with the class of ellipses which carry on $\eta$ up to $\xi$. Just observe that the other class of ellipses will not be useful for our purposes.

We notice that in the previous analysis the plane $\langle P, B\rangle=1$ intersect 
the cone $\mathcal{C}^{+}$and $A-B \in \mathcal{C}_{\text {ext }}^{+}$determine two singularities on the projective line. Fortunately, we do not need to care about. In fact, the optimal control given by the $P M P$ consider $u_{*}(t)=1$ or $u_{*}(t)=-\langle P(t), B\rangle$ or combinations of these controls.

2. $A-B \in \mathcal{C}_{\text {int }}^{+}$and $A+B \in \mathcal{C}_{\text {ext }}^{+}$

According to Remark 4.1, this situation is analogously to the case 1 .

3. $A+B, A-B \in \mathcal{C}_{\text {int }}^{+}$

a) If $\zeta_{0}, \xi \in \operatorname{cl}(U)$ the projection of any ellipse inside of $U$ starting on $\zeta_{0}$ and ending in $\xi$ will do the job

b) The case $\zeta_{0} \in \operatorname{cl}(U)$ and $\xi \in L$ is analogous to 1.2 (b)

c) The case $\zeta_{0} \in L$ and $\xi \in \operatorname{cl}(L) \cup U$ is analogous to 1.3 (a) and (b).

Suppose the plane $\langle P, B\rangle=-1$ intersect the cone $C^{+}$.

The optimal paths coming from the PMP just consider $u_{*}=-1$ or $u_{*}=\varphi(t)$. We define $L$ and $U$

$$
L=\left\{Z \in \mathcal{C}^{+} \mid-1<\langle Z, B\rangle<0\right\}, U=\left\{Z \in \mathcal{C}^{+} \mid\langle Z, B\rangle<-1\right\} .
$$

As before, the analyze follows by considering the cases

1. $A+B \in \mathcal{C}_{\text {int }}^{+}$and $A-B \in \mathcal{C}_{\text {ext }}^{+}$

In this situation, the synthesis of the extremals should be realized taking in account the singularities of $A-B$ which determine the regions $R_{1}=$ $\pi^{-1}\left(q_{-1}^{-}, q_{-1}^{+}\right]$and $R_{2}=\pi^{-1}\left(\mathbf{P}^{1} \backslash R_{1}\right)$.

1.1 If $p$ and $q$ lies in the same region, the analysis is analogously to the case $\langle P, B\rangle=1$, (1.).

1.2 If $p$ and $q$ lies in different regions, for instance, $p \in R_{1}$ and $q \in R_{2}$. Recall that $q_{-1}^{-}$is an attractor of $A-B$, in particular it is not possible to cross $R_{2}$ from $R_{1}$ with $u=-1$.

a) Let $\zeta_{0} \in \operatorname{cl}(L)$ and $\xi \in \operatorname{cl}(L)$. We just reach $q$ from $p$ through the projection of the differential equation determined by the optimal control $\varphi(t)=-\langle P(t), B\rangle \in(-1,1)$ with initial condition $\zeta_{0}$ and ending point $\xi$.

b) If $\zeta_{0} \in \operatorname{cl}(L)$ and $\xi \in U$ we start with $u=-\langle P(t), B\rangle$ moving up to the point $\xi_{0}$ : the intersection between plane $\langle P, B\rangle=-1$ in $R_{2}$ and the semi-hyperbola $\hbar_{\xi}^{-}$through $\xi$. So, the projection of $\hbar_{\xi}^{-} \circ \beta^{\varphi}$ send $p$ to $q$.

c) The case $\zeta_{0} \in U$ and $\xi \in R_{2}$ is not possible. Actually, we can not leave $R_{1}$.

2. $A-B \in \mathcal{C}_{\text {int }}^{+}$and $A+B \in \mathcal{C}_{\text {ext }}^{+}$

This case is analogously to the case (1.) taking care of the singularities of $A+B$. 
3. $A+B, A-B \in \mathcal{C}_{\text {int }}^{+}$

Analogous to the case (1.) of $\langle P, B\rangle=1$.

\subsubsection{Case 2. $\langle P, B\rangle=0$ is tangent to $\mathcal{C}^{+}$}

In this subsection we consider two cases

1. $B \in \mathcal{C}^{+}$and $A \in \mathbf{s l}(2)$. Without lost of generality we assume $A+B \in \mathcal{C}_{\text {int }}^{+}$. In particular, we get $A-B \in \mathcal{C}_{\text {ext }}^{+}$.

Assume the plane $\langle P, B\rangle=1$ intersect the cone $C^{+}$.

Figure 4.3: Intersection of $\langle P, B\rangle=1$ with $\langle P, A-B\rangle=0$ on $\mathcal{C}^{+}$

We denote by

$$
L=\left\{Z \in \mathcal{C}^{+} \mid 0<\langle Z, B\rangle<1\right\}, U=\left\{Z \in \mathcal{C}^{+} \mid\langle Z, B\rangle>1\right\}
$$

see Figure 4.3.

We begin the analysis when the fibers of both $p$ and $q$ intersect the same region. For instance, if $\pi^{-1}(p), \pi^{-1}(q)$ intersect $L$. We have two possibilities. The first one consider the projection of the solution with initial condition $\zeta_{0} \in \pi^{-1}(p)$ and ending state $\xi \in \pi^{-1}(q)$, through the adjoint differential equation determined by $u=\varphi$ inside of $L$. If we need to scape from $L$, then starting on $\zeta_{0}$ we move first under the influence of $u=\varphi$ up to $\xi_{0}$ : the intersection between the $\varphi$-integral curve and the plane $\langle P, B\rangle=1$. From the new initial condition $\xi_{0}$, we move on the region $U$ 
through the ellipse $\varepsilon^{+}$which intersect the plane $\langle P, B\rangle=1$ at the point $\xi_{1}$. From $\xi_{1}$ we enter inside of $L$ by a $\beta^{\varphi}$ trajectory up to reach the line $\pi^{-1}(q)$. Therefore, by an appropriated projection of the curve $\beta^{\varphi} \circ \varepsilon^{+} \circ \beta^{\varphi}$ we obtain the desired extremal on the projective line.

The case $\pi^{-1}(p), \pi^{-1}(q) \in \operatorname{cl}(U)$ is analogous.

Finally, if the fibers of both $p$ and $q$ intersect different regions. For instance, if $\pi^{-1}(p)$ intersect $L$, and $\pi^{-1}(q)$ intersect $U$, the analysis is similar to the previous cases. In fact, the extremals are given by the projection of $\varepsilon^{+} \circ \beta^{\varphi}$ or $\beta^{\varphi} \circ \varepsilon^{+}$.

Assume now the plane $\langle P, B\rangle=-1$ intersect the cone $C^{+}$

This situation is not possible. As before

$$
L=\left\{Z \in \mathcal{C}^{+} \mid-1<\langle Z, B\rangle<0\right\}, U=\left\{Z \in \mathcal{C}^{+} \mid\langle Z, B\rangle<-1\right\} .
$$

According to the $P M P$, on the region $L$ we always need to use the control $u=-1$. However, the line $\pi^{-1}\left(q_{-1}^{-}\right)$is an attractor of the adjoint equation. So, the trajectory will never leave $L$ and its projection will remains in $\left(q_{-1}^{+}, q_{-1}^{-}\right)$.

2. For $-B \in \mathcal{C}^{+}$and $A \in \mathbf{s l}(2)$ the synthesis follows in the same way as (1.) when $\langle P, B\rangle=-1$. But, $\langle P, B\rangle=1$ it is not possible.

\subsubsection{Case 3. $\langle P, B\rangle=0$ divides $\mathcal{C}^{+}$in two regions.}

In this subsection $B \in \mathcal{C}_{e x t}^{+}$. The planes $\langle P, B\rangle= \pm 1$ give rise to three regions in $\mathcal{C}^{+}$: the Left, Medium and Right regions. And $M$ has two connected components. Precisely,

$$
L=\left\{Z \in \mathcal{C}^{+} \mid \quad\langle Z, B\rangle \leq-1\right\}, M=\left\{Z \in \mathcal{C}^{+} \mid \quad-1 \leq\langle Z, B\rangle \leq 1\right\},
$$

and

$$
R=\left\{Z \in \mathcal{C}^{+} \mid 1 \leq\langle Z, B\rangle\right\},
$$

see Figure 4.1. In terms of those regions and according to the $P M P$, the synthesis states:

1. $A+B$ and $A-B$ belong to $\mathcal{C}_{\text {int }}^{+}$

a) The fiber of $p$ and $q$ intersect the same region. Suppose, this region is $R$. We have two different kind of dynamics

$$
\varepsilon^{+} \text {or } \varepsilon^{+} \circ \beta^{\varphi} \circ \varepsilon^{-} \circ \beta^{\varphi} \circ \varepsilon^{+}
$$


If both fibers meet $L$ or the same connected component of $M$ the synthesis is analogous. Assume, $\pi^{-1}(p)$ and $\pi^{-1}(q)$ belong two the different connected components of $M$, the possible dynamics are

$$
\beta^{\varphi} \circ \varepsilon^{-} \circ \beta^{\varphi} \text { or } \beta^{\varphi} \circ \varepsilon^{+} \circ \beta^{\varphi}
$$

Therefore, the projections of these classes of curves give us the extremals on $\mathbf{P}^{1}$.

b) If the fibers intersects two any different regions, the case (a) give the way to compute the extremals.

2. If $A+B$ or $A-B$ belong to $\mathcal{C}_{i n t}^{+}$, but not both.

Assume $A+B \in \mathcal{C}_{\text {int }}^{+}$and $A-B \in \mathcal{C}_{\text {ext }}^{+}$. In principle, we have two situations

2.1. The plane $\langle P, A-B\rangle=0$ intersects $\langle P, B\rangle=-1$ and $\langle P, B\rangle=1$ on $\mathcal{C}^{+}$.

Assume $\pi^{-1}\left(q_{-1}^{-}\right)$meets $R$ and $\pi^{-1}\left(q_{-1}^{+}\right)$meets $L$. According to the $P M P$, this situation is not possible. In fact, starting from a state in $R$ we can not reach any element of $L$.

2.2. The planes $\langle P, A-B\rangle=0,\langle P, B\rangle=-1$ and $\langle P, B\rangle=1$ are parallels.

This case is also not possible. Actually, under these hypothesis it follows that $\langle P, A\rangle=0$. Then, the matrices $A, B$ and $A-B$ are linear dependent which is a contradiction.

Summarizing, in the controllable case we have

Theorem 4.2. Assume that the projected control system (1.3) is controllable on $\mathbf{P}^{1}$. Given any two arbitrary points $p$ and $q$ on the real projective line, there exists an optimal control $u_{p, q}$ steering $p$ to $q$, minimizing the functional $J$ (1.2). The synthesis of $u_{p, q}$ is given through the Pontryagin Maximum Principle, as follows

$$
J\left(u_{p, q}\right)=\min \{J(u): u \in \mathcal{U} \text { determines an extremal }\}
$$

Next, we give some examples in which the projected system (1.3) is controllable.

Example 4.3. Let $\Sigma$ be determined by $A=\frac{H}{2}+2 R$ and $B=H+2 R$, see (2.1). Since, the segment $A+u B$ with $-1 \leq u \leq 1$, intercepts $\mathcal{C}_{\text {int }}^{+}$ the system is controllable. IN fact, $B, A+B \in \mathcal{C}_{\text {int }}^{+}$belong to $\mathcal{C}_{\text {int }}^{+}$and $A-B \in \mathcal{C}_{\text {ext }}^{+}$. Since the plane $\langle P, B\rangle=1$ meet the cone. This example corresponds to the Case 1, (1.). 
Example 4.4. Let $\Sigma$ be determined by $B=R-H \in \mathcal{C}^{+}$and $A=R$, see (2.1). Since, $A \in \mathcal{C}_{\text {int }}^{+}$the system is controllable, (4.1). On the other hand, $A+B \in \mathcal{C}_{\text {int }}^{+}$and $A-B \in \mathcal{C}_{\text {ext }}^{+}$and the plane $\langle P, B\rangle=1$ meets $\mathcal{C}^{+}$. So, this is an example of Case 2, (1).

Example 4.5. Consider $\Sigma$ defined by $B=H \in \mathcal{C}_{\text {ext }}^{+}$and $A=2 R$, see (2.1). Thus, $A+B \in \mathcal{C}_{\text {int }}^{+}$and $A-B \in \mathcal{C}_{\text {int }}^{+}$. If $-1 \leq u \leq 1$ the segment $A+u B$ intercepts $\mathcal{C}_{\text {int }}^{+}$then, the system is controllable. This situation correspond to Case 3, (1).

\subsection{The Non Controllable Case}

In this Section do not assume the controllability property of the projected system on the projective real line. The synthesis is established by considering the existence of two control sets of $\mathbf{P} \Sigma$ on $\mathbf{P}^{1}$.

Before proceeding we need the notion of control set (see [4], [11], [12]).

Definition 4.6. $A$ set $D \subset \mathbf{P}^{1}$ is called a control set of $\mathbf{P} \Sigma$ if for each $p \in D$

i) $D \subset \operatorname{cl} \mathbf{S}(p)$

ii) there exists a control $u \in \mathcal{U}$ with $\chi(t, p, u) \in D$ for all $t \geq 0$, and

iii) with respect to the set inclusion, $D$ is maximal with the properties (i) and (ii).

As usual, cl $M$ denotes the closure of the set $M$, the positive orbit $\mathbf{S}(p)$ is the action of the semigroup of the angle system on the state $p \in \mathbf{P}^{1}$ and $\chi(t, p, u)$ is the solution of the projected system with initial condition $p$ and control $u$. A main control set is a control set with nonvoid interior.

Since we assume that (1.1) satisfy the Lie algebra rank condition, the semigroup $\mathbf{S}$ of the bilinear control system is a proper subsemigroup of Sl (2) with int $\mathbf{S} \neq \emptyset$, see [11], [12]. Then, Proposition 3.1 in [1], shows that there are exactly two control sets on $\mathbf{P}^{1}$, denoted by $I^{ \pm}$. They satisfy the following properties:

i) $I^{-} \cap I^{+}=\emptyset, I^{-}$is closed and $I^{+}$is open

ii) $I^{-}$is invariant, i.e., $\mathbf{S}(p) \subset I^{-}$for all $p \in I^{-}$. On the other hand, $I^{+}$ is $\mathbf{S}^{-1}$-invariant 
iii) If $g \in \mathbf{S}$ is diagonalizable then its attractor belongs to $I^{-}$and its repeller is an element of the closure of $I^{+}$

As before, we consider matrices $A, B$ with $\operatorname{det}[A, B] \neq 0$. If $\mathbf{P} \Sigma$ is not controllable on $\mathbf{P}^{1}$ then $A+B$ and $A-B$ must be outside of $\mathcal{C}_{\text {int }}^{+}$. In the sequel, we just analyze the case, $B \in \mathcal{C}_{\text {ext }}^{+}$

From a dynamic point of view, the planes $\langle P, A+B\rangle=0$ and $\langle P, A-$ $B\rangle=0$ determine the control sets on $\mathbf{P}^{1}$, as follows

$$
I^{-}=\left[q_{+1}^{-}, q_{-1}^{-}\right], I^{+}=\left(q_{+1}^{+}, q_{-1}^{+}\right) .
$$

where the boundary point of the intervals are the singularities of $A+B$ and $A-B$ as explained above.

From the optimality point of view, the planes $\langle P, B\rangle= \pm 1$ give rise to the regions $L, M$ and $R$ in $\mathcal{C}^{+}$, and four intersection points on $\mathbf{P}^{1}$. All together determines the following eight regions on the projective line, which we describe in as in the Figure (4.4):

$$
\begin{aligned}
& \Delta_{1}=I^{-}, \Delta_{2}=\left(q_{-1}^{-}, q_{\langle P, B\rangle=1}^{2}\right], \Delta_{3}=\left(q_{\langle P, B\rangle=1}^{2}, q_{\langle P, B\rangle=-1}^{2}\right], \\
& \Delta_{4}=\left(q_{\langle P, B\rangle=-1}^{2}, q_{+1}^{+}\right], \Delta_{5}=I^{+}, \Delta_{6}=\left(q_{-1}^{+}, q_{\langle P, B\rangle=-1}^{1}\right], \\
& \Delta_{7}=\left(q_{\langle P, B\rangle=-1}^{1}, q_{\langle P, B\rangle=1}^{1}\right], \Delta_{8}=\left(q_{\langle P, B\rangle=1}^{1}, q_{+1}^{-}\right) .
\end{aligned}
$$

Figure 4.4: Dynamic on $\mathbf{P}^{1}$

In terms of those regions and according to the $P M P$, we must apply the control $u=-1$ on $L, u=\varphi(t)=-\langle P(t), B\rangle$ on $M$ and $u=1$ inside of $R$. For $p<q$, we get in the table 1 for the possible trajectories on the cone $\mathcal{C}^{+}$ 
Here $p \stackrel{\text { ext }}{\longrightarrow} q$ denotes an extremal path on $\mathbf{P}^{1}$ starting on $p$ and ending on $q$ with $p<q$.

As a consequence, there exists an extremal path starting on any point of $I^{+}$and ending on an arbitrary point in the invariant control set $I^{-}$. The optimal path is constructed using bang controls as well as controls with values in the interior of $[-1,1]$. However, this is not the case in the other direction.

Next, we illustrate an example in the noncontrollable case

Example 4.7. Consider $\Sigma$ with the basis vectors $A=S$ and $B=H \in \mathcal{C}_{e x t}^{+}$, see (2.1). The system $\Sigma$ satisfies LARC but is not controllable. The plane $B^{\perp}$ splits $\mathcal{C}^{+}$in two regions conen $\left\{z_{1}>0\right\}$, and cone $\cap\left\{z_{1}<0\right\}$ and both Planes $\langle P, A \pm B\rangle=0$ intersect to $\langle P, B\rangle= \pm 1$ on $\mathcal{C}^{+}$.

Since, $A+B$ as well as $A-B$ belong to $\mathcal{C}_{\text {ext }}^{+}$. Then the control sets are given by

$$
\begin{gathered}
I^{+}=\left(\frac{(1,-1-\sqrt{2})}{\|(1,-1-\sqrt{2})\|}, \frac{(1,1-\sqrt{2})}{\|(1,1-\sqrt{2})\|}\right) \text { and } \\
I^{-}=\left[\frac{(1, \sqrt{2}-1)}{\|(1, \sqrt{2}-1)\|}, \frac{(1,1+\sqrt{2})}{\|(1,1+\sqrt{2})\|}\right]
\end{gathered}
$$

where $\|\cdot\|$ denotes the usual norm for vectors in $\mathbf{R}^{2}$. (see Figure 4.4). 
Let us $p$, for instance, the middle point of the $I^{+}$. There exists a extremal path connecting any two point $p \in I^{+}$and $q \in I^{-}$starting on $p$, for instance $p$ the middle point of the interval $I^{+}$with the point

$q=\left(\frac{2}{\sqrt{7-2 \sqrt{2}}}, \frac{\sqrt{2}-1}{\sqrt{7-2 \sqrt{2}}}\right) \in$ Int $I^{-}$. This extremal path is constructed, according to the PMP using the concatenation of the controls bang-interiorbang.

\section{References}

[1] Ayala V, and San Martin, L.A.B. , Controllability of two-dimensional bilinear system: restricted controls, discrete-time, Proyecciones, 18, pp. 207-223, (1994).

[2] Ayala V., J.C. Rodríguez and L.A.B. San Martin, Optimality on Homogeneous spaces, and the angle system associated with a bilinear control system, SIAM J. Control Optim., 48, (4), pp. 2636-2650, (2009).

[3] Braga B. C. J., Gonçalves J.R., O. do Rocio and L.A.B. San Martin, Controllability of two-dimensional bilinear systems, Proyecciones, 15, pp. 111-139, (1996).

[4] Colonius F. and Kliemann W., Dynamics and Control, Birkh äuser, (1999).

[5] D. Kirschner, S. Lenhart, S. Serbin, Optimal control of Chemoterapy of HIV , J. Math. Biol., 35, pp. 775-792, (1997).

[6] Jacobson D. M., Extensions of linear quadratic control theory, Springer-Verlag, New York, (1980).

[7] Jurdjevic V., Geometric control theory, Cambridge University Press, New York, (1997).

[8] Ledzewick U., Shättler H., Optimal controls for a two compartment model for cancer Chemoterapy with quadratic objective, Proceedings of Controlo 2002, Aveiro, Portugal, pp. 241-246, (2002).

[9] Sachkov Y. L.: Control Theory on Lie groups, SISSA, (2006). 
[10] Sagle A. and Walde R.: Introduction to Lie group and Lie algebras, Pure and Applied Mathematics, Editors Eilenberg, New York,(1973).

[11] San Martin L. A. B. and Tonelli P. A., Semigroup actions on Homogeneous spaces, Semigroup Forum, 50, pp. 59-88, (1995).

[12] San Martin L.A.B., Invariant control set on flag manifolds, Math. Control, Signals and systems, 6, (1993), 41-61.

[13] Sussmann H., Lie Brackets, Real Analicity and Geometric Control, Progress in Mathematics, Differential Geometric Control Theory, Proceedings of the conference held at Michigan Technological University, 27, pp. 1-116, (1982).

\section{Víctor Ayala}

Departamento de Matemáticas

Universidad Católica del Norte

Casilla 1280

Antofagasta - Chile

e-mail : vayala@ucn.cl

\section{J. C. Rodríguez}

Departamento de Matemáticas

Universidad Católica del Norte

Casilla 1280

Antofagasta - Chile

e-mail : jrodriguez@ucn.cl

and

\section{A. B. San Martín}

Instituto de Matemática Estatística e Ciências da Computação

Universidade Estadual de Campinas

Cx. Postal 6065

13081-970 Campinas SP

Brasil 